\title{
Bullletin Board
}

New study revealed that administering targeted radiotherapy following aggressive surgery yields a 5 -year overall survival of $85 \%$ in pediatric patients suffering from a rare childhood brain tumor

\section{Good survival rates with aggressive surgery followed by radiotherapy in young patients with ependymoma}

A recent study yielded the highest ever recorded success rates in treating ependymoma, showing aggressive surgery coupled with targeted radiotherapy to be an effective form of treatment.

Ependymoma, a rare type of glioma, affects 0.76 of 100,000 individuals aged $0-19$ years in the USA every year. With only a 50\% 5-year survival rate in the youngest patients, prognosis is especially poor.

Aiming to improve tumor control and assess treatment efficacy, researchers examined 153 children treated for localized ependymoma between 1997 and 2007. This investigation was an update and extension of previous findings published in 2004 and is the first to include children under the age of 3 years.

All participants underwent aggressive surgery; 125 patients underwent gross total resection with no evidence of residual tumor on MRI, while 17 patients underwent near total resection and 11 patients underwent subtotal resection. Most patients (131) then received a dosage level of 59.4 Gy of conformal radiotherapy, with the remainder (22) receiving 54.0 Gy. The children's ages ranged from 0.9 to 22.9 years, with a median age of 4.9 years. Treatment followup spanned from 0.4 to 10.4 years with a median follow-up of 5.3 years.

The results of the study, according to Dr Thomas Merchant, lead author and chief of the Division of Radiation Oncology at St Jude's Children's Hospital of Memphis, Tennessee, are the best ever reported for patients with ependymoma. "The eventfree survival of $77 \%$ at 7 years and overall survival of $85 \%$ at 7 years exceeded expectations from all reported series." Whereas other researchers have reported a 5-year event-free survival ranging from 41 to $58 \%$ and a 10-year event-free survival from 31 to $46 \%$ for patients, Merchant and his colleagues reported a 74 and $69 \%$ event-free survival, respectively.

As "local failure has been the greatest obstacle to improving overall survival in ependymoma," the researchers attribute improvements to increased local tumor control, in which younger patients show the largest gains. However, improvements in local control were offset by an increase in metastatic failures. As metastatic failures appeared to be more common in patients with anaplastic tumors, such patients may benefit from alternative approaches to treatment.

The finding of $85 \% 5$-year overall survival in this series was in contrast to previous findings of $65 \%$ overall survival for treatment with chemotherapy alone. However, further research is required to compare the efficacy of chemotherapy with radiotherapy following surgery in order to reach a consensus regarding optimal treatment approach. The authors conclude that future trials may wish to utilize treatment stratification on the basis of sex and age.

Merchant is optimistic that "newer methods of radiotherapy delivery promise further reductions in the dose (delivered) to healthy tissue," which will minimize radiotherapy-related side effects.

Source: Merchant TE, Li C, Xiong X,

Kun LE, Boop FA, Sanford RA: Conformal

radiotherapy after surgery for pediatric

ependymoma: a prospective study. Lancet Oncol. DOI:10.1016/S1470-2045(08)70342-5 (2009)

(Epub ahead of print). in the news...

- Good survival rates with aggressive surgery followed by radiotherapy in young patients with ependymoma

ain brief

- Formula may create universal standard for measuring kidney function in children

- Genetic link to leukemia treatment response

Exon skipping drug may be a potential treatment for Duchenne muscular dystrophy 


\section{in brief.}

Ophthalmologic findings in children with sensorineural hearing loss.

Sharma A, Ruscetta MN, Chi DH: Arch.

Otolaryngol. Head Neck Surg. 135(2),

119-123 (2009).

Approximately one to three children per 1000 live births have at least moderate sensorineural hearing loss (SNHL) and approximately four children per 10,000 live births have profound SNHL. According to this study's findings, just under a fifth $(21.7 \%)$ of these children will have ocular disorders as well. The study examined 226 patients with SNHL seen at a children's hospital between 2000 and 2007 for ophthalmologic anomalies. Researchers found that 49 patients $(21.7 \%)$ had an ophthalmologic abnormality; 23 (10.2\%) had refractive errors and $29(12.8 \%)$ had nonrefractive errors. The cause of SNHL was syndromic in 11 patients (4.9\%), five $(2.2 \%)$ of whom had syndromes with related ophthalmologic abnormalities. Patients with biallelic GJB2 mutations had a significantly lower prevalence (3.7\%) of ophthalmologic abnormalities than children with normal GJB2 $(20.8 \%)$. This finding is consistent with previous literature suggesting that GJB2 mutations lead to SNHL but not to additional abnormalities or syndromes. No statistically significant differences for ocular abnormality were found based on severity or laterality of hearing loss. These findings indicate that routine ophthalmologic examination may be beneficial in all children with SNHL.

Hyperglycemia results from b-cell dysfunction in critically ill children with respiratory and cardiovascular failure: a prospective observational study. Preissig CM, Rigby MR: Critical Care DOl:10.1186/cc7732 (2009) (In Press). Describes how both peripheral insulin resistance and primary $\beta$-cell dysfunction can cause critical illness hypergylcemia $(\mathrm{ClH})$ in children. The authors state that $\mathrm{CIH}$ is highly prevalent and estimate that approximately $20 \%$ of admissions to their intensive care unit develop the condition. The study followed 41 children receiving intensive care treatment and found that those with respiratory failure only had $\mathrm{ClH}$ caused by elevated insulin resistance. The other children with both respiratory and cardiovascular failure appeared to have $\mathrm{ClH}$ caused by primary $\beta$-cell dysfunction. The authors note that further studies are required to confirm if insulin treatment is effective in both subgroups of patients. The study concluded that patients with respiratory failure and cardiovascular failure also had more severe $\mathrm{ClH}$ than those with respiratory failure alone. They hope that this research will lead to the use of individualized glycemic goals and treatment strategies in children with life-threatening illness or injury.

A new formula may change assessment of kidney function in children and improve monitoring and treatment of pediatric patients with chronic kidney disease

\section{Formula may create universal}

\section{standard for measuring kidney function in children}

Researchers have recently devised a formula for measuring kidney function using parameters easily obtained in clinical settings, which may ultimately lead to better monitoring of young patients with abnormal kidney function.

The authors of this study aimed to improve current methods for measuring kidney function; prior means have been expensive and time-consuming for clinicians and children are exposed to radioactivity and subjected to repeated blood draws. As most existing formulas are singular to their institutions or hospitals, researchers sought to develop a universal standard for assessing kidney function.

The glomerular filtration rate (GFR), the rate at which fluid is filtered through the kidneys, has often served a useful indicator of kidney function and kidney disease progression. Although this has been difficult to measure in children, the Schwartz formula was originally devised in the 1970s for this purpose. Recent data suggest that this method overestimates GFR, and the authors aimed to improve the formula's accuracy.

\section{"....an accurate estimate is} crucial to properly treat children with acute and chronic kidney problems and the scale of this study makes its findings quite significant."

Using linear regression analysis to enhance precision, goodness of fit and accuracy, the new formula uses variables that can be quickly and easily obtained without the use of radioactivity or urine collection. By measuring height, gender, serum creatinine, blood urea nitrogen, and cystatin $\mathrm{C}$ (a ubiquitous protein that is well correlated with kidney function), blood tests performed in a clinical setting can generate an accurate estimate of a child's kidney function. A bedside calculation of $0.413 \times$ (height/serum creatinine) is a good approximation of the estimated GFR formula.

In a 1-year follow-up of a test set of 168 young patients with chronic kidney disease, researchers found the estimating formula to be superior to other recently published equations and comparable with more complicated tests. Principal investigator George Schwartz of University of Rochester Medical Center's Golisano Children's Hospital of Rochester, NY, USA commented that, "an accurate estimate is crucial to properly treat children with acute and chronic kidney problems and the scale of this study makes its findings quite significant." Such research may lead to fewer episodes of acute kidney injury in children and may also improve methods for adjusting fluids, and administering antibiotics and chemotherapy.

Based on data collected by Chronic Kidney Disease in Children (CKiD) study, the sample population consisted of 349 patients, aged $1-16$ years, from over 50 North American institutions. As the study focused on children suffering from chronic kidney disease, Schwartz notes that "it will also be important to determine if such a formula will apply to children and infants with higher level of kidney function so that any harm that might come from antibiotics, chemotherapeutic drugs and dehydration can be minimized in these populations as well."

Source: Schwartz GJ, Muñoz A, Schneider MF et al: New equations to estimate GFR in children with CKD. J. Am. Soc. Nephrol. DOI: 10.1681/ ASN.2008030287 (2009) (Epub ahead of print). 


\section{Genetic link to leukemia treatment response}

A new discovery could lead to individualized treatment approaches in children with acute lymphoblastic leukemia.

Scientists from St Jude Children's Research Hospital and the Children's Oncology Group (COG) have discovered over 100 genetic variations linked to leukemia treatment response.

Although treatment success rates for pediatric acute lymphoblastic leukemia (ALL) exceed $80 \%$, treatment response varies considerably between individuals. The authors of this study explored genetic contributions to therapy response, aiming to identify germline single-nucleotide polymorphisms (SNPs) associated with risk of minimal residual disease (MRD), the small number of leukemic cells surviving remission-induction therapy.

Researchers investigated 476,796 SNPs in 487 children from two groups treated for newly diagnosed ALL. Participants were enrolled between 1994 and 2006, with the last follow-up conducted in 2006.
Contrary to previous studies, researchers focused on inherited genomic variation in all cells, rather than specifically targeting the genes of leukemic cells.

The authors found 102 SNPs significantly associated with MRD in both cohorts, even after controlling for race, sex, leukocyte count at diagnosis, age and ALL subtype. A total of 21 of these 102 MRD-linked SNPs were also associated with leukemic relapse, and 21 SNPs were linked to the eradication of MRD with increased exposure to chemotherapy drugs. The researchers' findings may also warrant divergent approaches to treatment; ultimately, 63 of 102 SNPs were linked with early response, relapse, or drug disposition.

Researchers also observed five SNPs in the IL15 gene, which is responsible for leukemic cell multiplication and is also thought to protect tumors from certain chemotherapy drugs. The IL15 SNPs were linked to increased levels of IL15 in leukemia cells and an elevated risk of high MRD following induction therapy. Author Mary Relling commented that, "IL15 might also represent a new target for novel drugs that knock out its activity and improve the outcome of patients with high levels of this interleukin."

Jun Yang of St Jude Department of Pharmaceutical Sciences and the paper's first author comments that, "our genomewide approach to identifying such SNPs is useful for identifying genetic variations that can be used to predict treatment outcomes." Yang also adds that, "such variation may be factored into treatment decisions in the future by placing additional emphasis on optimizing drug delivery to overcome host genetic variation, in addition to the current emphasis on tumor genetic variation."

Source: Yang JJ, Cheng C, Yang W et al.:

Genome-wide interrogation of germline genetic

variation associated with treatment response in

childhood acute lymphoblastic leukemia. JAMA 301(4), 393-403 (2009).

\section{Exon-skipping drug may be a potential treatment for Duchenne muscular dystrophy}

\begin{abstract}
Duchenne muscular dystrophy (DMD) is a form of muscular dystrophy that affects approximately one in every 3500 boys worldwide. It is also caused by a mutation in the dystrophin gene, the product of which plays a key role in muscle fiber function. The disease progresses rapidly, resulting in complete paralysis and eventually death by the age of 30 years.
\end{abstract}

\footnotetext{
"We are very pleased to begin the systemic evaluation of our exon-skipping drug - AVI-4658 - for the treatment of DMD..."
}

A clinical trial evaluating the systemic delivery of AVI- 4658 for the treatment of DMD has recently been initiated.

AVI-4658 uses an Exon-Skipping Pre-RNA Interference Technology (ESPRIT) developed by AVI BioPharma, Incorporated. Studies in animal models have shown that ESPRIT technology is able to selectively bypass defective exons. It is thought that as AVI-4658 skips exon 51 , a truncated but functional form of the dystrophin protein is produced. As a result, normal levels of dystrophin are restored, thus limiting further decline in muscle function.

Results from a Phase I study announced earlier this year demonstrated that injecting AVI-4658 into the muscles of DMD patients induced the production of dystrophin in a dose-responsive manner. In addition, the drug was well tolerated and no significant adverse events were observed.

The current trial will enroll 16 ambulatory boys suffering from DMD. It is an open-label, 12-week safety trial and will assess the pharmacokinetics and efficacy of AVI-4658 at doses between 0.5 and $4.0 \mathrm{mg} / \mathrm{kg}$.
"We are very pleased to begin the systemic evaluation of our exon-skipping drug - AVI-4658 - for the treatment of DMD," said Stephen Shrewsbury, Chief Medical Officer and Senior Vice President, Clinical and Regulatory Affairs of AVI BioPharma. "We believe that this trial will build significantly on the data generated by the successful recent trial evaluating intramuscular administration of the same drug in DMD boys."

Currently, there is no cure for DMD and therapies existing are aimed at treating the symptoms. Several other therapies are presently under investigation. It is hoped that AVI-4658 will prove effective in treating this devastating disease.

Source: Duchenne muscular dystrophy - AVI4658 recommended for orphan drug status in EU www.medicalnewstoday.com/articles/126169.php 